\title{
PRODUCTION OF POROUS TI5A12.5Fe ALLOY VIA PRESSURELESS SPARK PLASMA SINTERING
}

\author{
R. Yamanoglu, ${ }^{a,{ }^{*}}$ N. Gulsoy, ${ }^{\mathrm{b}}$ E. A. Olevsky, ${ }^{\mathrm{c}}$ H. O. Gulsoy ${ }^{\mathrm{d}}$
}

a Kocaeli University, Engineering Faculty, Metallurgical and Materials Engineering Department, Kocaeli, Turkey

${ }^{\mathrm{b}}$ Marmara University, Faculty of Arts and Sciences, Biology Department, Istanbul, Turkey

${ }^{c}$ San Diego State University, College of Engineering, Mechanical Engineering Department, San Diego, CA, USA

d Marmara University, Technology Faculty, Metallurgical and Materials Engineering Department, Istanbul, Turkey

\begin{abstract}
Porous Ti5A12.5Fe alloy was successfully fabricated using the spark plasma sintering technique. Experiments were performed within a temperature range of between 750 and 850 ${ }^{\circ} \mathrm{C}$. The microstructure, compression and biocompatibility properties of the porous samples were investigated. Sintered samples were immersed for different times in a simulated body fluid with elemental concentrations comparable to those of human blood plasma. Porous sample was also implanted in fibroblast culture for biocompatibility evaluation. The compressive strength of the porous samples was found to be mainly dependent on sintering temperature, and the porous compacts exhibiting good biocompatibility properties.
\end{abstract}

Keywords: Titanium alloys; Compression test; Microstructure; Biocompatibility

* Corresponding author. Tel.: +90 507 9260532; e-mail: ryamanoglu@kocaeli.edu.tr 


\section{Introduction}

Metallic materials are suitable for hard tissue applications such as artificial hip joints [1]. Among the various metallic biomaterials available, titanium and its alloys are preferred for orthopedic and dental implants due to their excellent biocompatibility, osseointegration properties, and high strength-to-weight ratio [2,3]. One of the major problems related to the use of metallic biomaterials in orthopedic surgery is the mismatch in Young's modulus between bone and metallic material [1,4]. Porous metallic materials are currently attracting increased research attention as a method with which to reduce such mechanical mismatches and also to achieve stable long-term fixation by means of full bone ingrowth [5]. However, the porous material used must meet certain requirements. For instance, the pores should be open, sufficiently large, and form through channels connected with one another, while the porous material should also exhibit suitable mechanical properties [6].

Porous titanium parts can be prepared using a number of different methods, such as traditional powder metallurgy, direct laser metal sintering, electro discharge sintering, multiple coating techniques, and sequential freeze casting. However, in most of these techniques it is difficult to effectively control the porous structure produced, resulting in poor biomechanical properties and low osteoconductivity [7]. In contrast, the use of powder metallurgical methods enables the production of porous materials with suitable properties. For example, Oh et al. [8] successfully fabricated porous Ti compacts via powder sintering, with a porosity range from 5 to $37 \%$.

The most common titanium-based implant materials used for commercial purposes are pure titanium and Ti6Al4V alloy [9]. Pure titanium is typically preferred for applications in which corrosion resistance is of critical importance, such as dental implants. However, when the mechanical properties of the material are the main focus, Ti6Al4V alloy is widely considered one of the best materials for use in medicine due to its mechanical behavior, chemical stability, and easy control of its microstructure [10]. Recent studies examining such biomedical materials concerns the toxic effects of aluminum and vanadium. Especially vanadium in the alloy may cause serious problems to the human body. The toxicity of vanadium is known and can be particularly severe when the implant is fractured under conditions of fatigue [11]. Researchers now attempting to identify safer titanium-based 
biomaterials. For example, Ti5Al2.5Fe has similar properties to those of Ti6Al4V and was developed in response to concerns over potential vanadium cytotoxicity [12].

Spark plasma sintering (SPS), also known as the field assisted sintering technique (FAST) or pulsed electric current assisted sintering (PECAS), is a novel sintering method able to produce high-density components at relatively low process temperatures and short holding times compared with conventional sintering techniques. SPS has been employed to fabricate fulldensity compacts from a number of traditionally difficult to sinter materials in the fields of metals, ceramics and composites [13-14].

During the SPS process, a pulsed DC current and uniaxial pressure are applied to a green body in a graphite die; activation of the particles is achieved by the application of an electrical discharge. For electrically conductive materials, heating occurs mainly due to the Joule effect. Although the occurrence of plasma discharge in proximity to the surface particles remains under debate, it is widely accepted that an electrical discharge is produced on a microscopic level. This effect may in turn result in a number of phenomena that enhance densification, such as surface particle activation or local melting and evaporation on the surface of the particles, which induce neck formation. The presence of the electrical field accelerates diffusion due to the enhanced ion migration speed. Densification can be achieved rapidly and, therefore, this process can be employed to control grain growth by using sintering times shorter than those typical of conventional processes such as hot pressing or hot isostatic pressing [15-16]. Pressure-assisted SPS has previously been used in the production of various different titanium alloys. For example, Ibrahim et al. [17] used SPS to sinter both pure titanium and Ti5Mn alloys of varying porosities, with $\mathrm{NH}_{4} \mathrm{HCO}_{3}$ used as a space holder and $\mathrm{TiH}_{2}$ as the foaming agent.

However, although Ti5Al2.5Fe alloy has high strength and is thus expected to be widely used as an implant material there is limited research on its microstructural, mechanical and biocompatibility properties. The present study was therefore carried out in order to examine the production, mechanical and biocompatibility properties of the alloy processed via pressureless spark plasma sintering. 


\section{Experimental}

In the present study, a pressureless spark plasma sintering method was employed for the production of porous Ti5Al2.5Fe alloy, with processing accomplished using a Dr Sinter Lab 515S system (SPS Syntex Inc.). In order to achieve pressureless conditions during SPS processing, the regular shape design was modified based on Bradbury et al. [18]. T-shape rather than cylindrical punches were used, composed of the same graphite material used in die fabrication. A working space was obtained between the punch faces within the standard SPS die, which experienced no external load during processing, as seen in Figure 1a. Die and punch outer diameter was $30 \mathrm{~mm}$, and interior die and inner punch diameters 10.4 and $10 \mathrm{~mm}$, respectively. Minimum pressure was applied in order to supply sufficient contact resistance through the punch exterior and die wall interior. Directed current routing was accomplished via the placement of graphite paper spacers pressed between the die and punch faces.

Ti-5Al-2.5Fe pre-alloyed powders were used as the raw material with which to produce the porous structure. Figure 1b, which shows a scanning electron microscopy (SEM) image of a powder, reveals that the particles exhibited a spherical morphology with no satellite formation. The powders were subjected to pressureless spark plasma sintering (PSPS) with a heating rate of $100{ }^{\circ} \mathrm{C} \min ^{-1}$, and were held at 750,800 and $850{ }^{\circ} \mathrm{C}$ for $5 \mathrm{~min}$ in a vacuum. Minimal contact pressure was maintained at $5 \mathrm{MPa}$ throughout the process, but was completely supported by the die. DC pulse timing was held constant at 12 pulses on and 2 pulses off, with a pulse duration of $\sim 3.3 \mathrm{~ms}$; the temperature was measured using a K-type thermocouple mounted into the graphite die wall. Sintered densities of the porous compacts were determined using a conventional gravimetric method. Compression properties of the produced porous Ti5Al2.5Fe alloy were carried out using an Instron 5982 Universal Testing Machine equipped with a $100 \mathrm{kN}$ load cell. Compressive data were obtained in the Bluhill 2 DAQ software package. A slow strain rate $\left(0.5 \mathrm{~mm} \mathrm{~min}^{-1}\right)$ was applied in all tests.

Additional cylindrical porous compacts were then prepared metallographically in rough sand and rinsed ultrasonically in acetone, absolute alcohol and deionized water in turn five times for biocompatibility tests. The porous samples were dried in an oven at $70{ }^{\circ} \mathrm{C}$ prior to SBF analysis, followed by immersion in sealed test tubes containing $20 \mathrm{ml} \mathrm{SBF}$ for 7, 14 or 21 days. A more detailed description of the employed SBF preparation process can be found in [19]. Experiments were performed in a laboratory water bath maintained at a constant 
temperature of $37.5{ }^{\circ} \mathrm{C}$ and subjected to a continuous vibrating motion in order to help maintain a uniform ion concentration. After immersion in SBF for the selected period, the compacts were retrieved, gently rinsed with distilled water, and dried at $45{ }^{\circ} \mathrm{C}$ for 1 day. After drying, the samples were sputter-coated with $\mathrm{Au}$ and the surfaces examined via scanning electron microscopy (JEOL JSM-6060) using a voltage of $15 \mathrm{kV}$.

\section{Results and discussion}

The final compact dimensions were a length of $10 \mathrm{~mm}$ and a diameter of $15 \mathrm{~mm}$; macro and SEM images of the produced porous compacts are shown in Figure 2. No considerable size and shape changes were seen due to the absence of any pressure application. The porosity ratio of the specimens decreased with increasing temperature, with the samples sintered at 750,800 and $850{ }^{\circ} \mathrm{C}$ exhibiting ratios of $29.1,28.7$ and $28.4 \%$, respectively. Increasing the sintering temperature thus caused a small decrease in compact porosity level. In biomedical parts, a higher degree of both bone growth into porous surfaces and body fluid transport through three-dimensional interconnected pore arrays are required. A number of studies have shown that the porosity level of titanium compacts considered suitable for bone replacement is around $30 \%$, a figure in accordance with the proposed optimal porosity value for the ingrowth of new-bone tissues [20]. Therefore, the porosity results recorded in the present study demonstrate the suitability of the tested compacts as implant materials. In powder metallurgy, the three stages of the sintering process are typically classified based on the neck size/particle size ratio (X/D). If the X/D ratio is smaller than 0.33 , between 0.33 and 0.5 , or higher than 0.5 , the process can be estimated as at the initial stage, intermediate stage, and final stage, respectively [21]. In the present study the (X/D) size ratio was normally affected by the sintering temperature, with samples sintered at 750,800 and $850{ }^{\circ} \mathrm{C}$ recording ratios of $0.27,0.29$, and 0.30 , respectively. Although these values are below 0.33 , particle bonding in the compacts was strong. The pulse voltage produced between connected particles during spark plasma sintering caused enhanced diffusion at the contact points (Figure 2b) of particles, resulting in enhanced mechanical properties. Figure $2 b$ also shows the dimple mode fracture region of these contact points.

The typical porous structure of metallic alloys can absorb large amounts of energy when exposed to compression deformation, with the maximum force generated by the porous structure always lower than the dense body under the given level of energy [22]. The value of the Young's Modulus can be decreased by producing high porosity levels. However, a porous 
structure also affects the material's mechanical properties. In the present study we also investigated the compression properties of the produced porous Ti5 $\mathrm{Al} 2.5 \mathrm{Fe}$ alloy. An image of a sample after the compression test is shown in Figure 3. The compressive strength of the porous compacts increased with increasing sintering temperature, with the sample sintered at $850{ }^{\circ} \mathrm{C}$ exhibiting a compressive strength of around $300 \mathrm{MPa}$. During the compression tests, deformation in the parts became non-uniform along the diagonal axes at $45^{\circ}$ to the loading direction, as indicated by the arrows in Figure 3. This shear band then caused a reduction in the load carrying capacity of the deforming compact during the compression test. Failure was completed by the separation of the bonded particles on the shear bands, starting from the corners of the cylindrical compact sample (Figure 3) [19].

SEM images of the samples placed in SBF for the three different periods are shown in Figures 4a, b, and c. Analysis of Figure 4a reveals that after 7 days immersion, a number of globular apatite particle islands had grown on the compact surface, with the quantity and size of the apatite particles gradually increasing with immersion time. After 21 days immersion, the surfaces of the samples were covered with large apatite particles. Subsequent EDS characterization of the tested samples revealed that the dominant elements present on the sample surfaces were $\mathrm{Na}, \mathrm{Cl}$ and $\mathrm{Ca}$ after immersion in SBF for all periods.

For the cell culture tests, a 3T3 rat fibroblast cell line was provided by American Type Culture Collection (Rockville, MD, USA) and was grown in a monolayer culture in Dulbecco's Modified Eagle's Medium-F12 (DMEM-F12; Biological Industries, Israel) supplemented with 10\% heat-inactivated fetal calf serum (Sigma Chemical Co., St Louis, Missouri). Following Trypan Blue exclusion assay, 3T3 cells were plated in six-well culture plates containing $5 \mathrm{ml}$ DMEM-F12 medium at a concentration of $1 \times 10^{5}$ cells/well. After attachment, compacts were placed in the wells. Cells were harvested at $96 \mathrm{~h}$, with the total cell number determined using an automated cell counter (Nucleocounter, Denmark). The Statistical Packages for the Social Sciences (SPSS) 17.0 statistical software program (SPSS, Inc., Chicago, IL, USA) was used for statistical analysis. Cell culture tests indicated that the produced porous $\mathrm{Ti} 5 \mathrm{~A} 12.5 \mathrm{Fe}$ compacts were biocompatible. According to the cell viability test the compact exposed to the pure culture medium (control group) exhibited $99 \%$ cell viability. In the porous compacts, cell viability was maintained at $88 \%$. When compared to the control group, the porous samples presented reasonable vitality. Figures $4 d$ and e show 
images of cells found on the sample surfaces after $96 \mathrm{~h}$ culture. All cells were well spread along the surfaces, as shown in Figure 4e, with some cell growth also observed.

4. Conclusion

We successfully prepared a porous Ti5Al2.5Fe alloy by a pressureless spark plasma sintering method. The pressure was supported by the graphite during the process to produce porous structure. Increasing sintering temperature caused a small decrease in compact porosity level and an extensive increase in compressive strength. After biocompatibity test, the sample showed reasonable vitality.

Acknowledgement

The support of The Scientific and Technological Research Council of Turkey - TUBITAK is greatly appreciated. This material is based on work supported by the US Department of Energy, Materials Sciences Division, under Award No. DE-SC0008581, through the Powder Technology Laboratory at the San Diego State University.

\section{References}

[1] G. He, M. Hagiwara, Bimodal structured Ti-based alloy with large elasticity and low Young's modulus, Mat Sci Eng C. 25 (2005) 290-295.

[2] J. B. Fogagnolo, A. V. Rodrigues, M. S. F. Lima, V. Amigo, R. Caram, A novel proposal to manipulate the properties of titanium parts by laser surface alloying, Scripta Mater. 68 (2013) 471-474.

[3] J. Wieding, T. Lindner, P. Bergschmidt, R. Bader, Biomedical stability of novel mechanically adapted open-porous titanium scaffolds in metatarsal bone defects of sheep, Biomaterials. 46 (2015) 35-47.

[4] N. Nomura, T. Kohama, I. H. Oh, S. Hanada, A. Chiba, M. Kanehira, K. Sasaki, Mechanical properties of porous $\mathrm{Ti}-15 \mathrm{Mo}-5 \mathrm{Zr}-3 \mathrm{Al}$ compacts prepared by powder sinteriing, Mat Sci Eng C. 25(3) (2005) 330-335. 
[5] X. Li, C. Wang, W. Zhang, Y. Li, Fabrication and characterization of porous Ti6A14V parts for biomedical applications using electron beam melting process, Mater Lett. 63 (2009) 403-405.

[6] A. P. Rubshtein, I. S. Trakhtenberg, E. B. Makarova, E. B. Triphonova, D. G. Bliznets, L. I. Yakovenkova, A. B. Vladimiroz, Porous material based on spongy titanium granules: Structure, mechanical properties, and osseointegration, Mat Sci Eng C. 35 (2014) 363-369.

[7] G. Jiang, Q. Li, C. Wang, J. Dong, G. He, Fabrication of graded porous titaniummagnesium composite for load-bearing biomedical applications, Mater Design. 67 (2015) 354-359.

[8] I. H. Oh, N. Nomura, N. Masahashi, S. Hanada, Mechanical properties of porous titanium compacts prepared by powder sintering, Scripta Mater. 49 (2003) 1197-1202.

[9] B. D. Ratner, A. S. Hoffman, F. J. Schoen, J. E. Lemons, Biomaterials Science, third ed., Elsevier, 2004.

[10] F. A. Shah, M. Trobos, P. Thomsen, A. Palmquist, Commercially pure titanium (cp-Ti) versus titanium alloy (Ti6Al4V) materials as bone anchored implants - Is ore truly better than the other?, Mat Sci Eng C. 62 (2016) 960-966.

[11] V. S. A. Challa, S. Mali, R. D. K. Misra, Reduced toxicity and superior cellular response of preosteoblasts to Ti-6Al-7Nb alloy and comparison with Ti-6Al-4V, J Biomed Mater Res Part A. 101 (2012) 2083-2089.

[12] R. P. Siqueira, H. R. Z. Sandim, A. O. F. Hayama, V. A. R. Henriques, Microstructural evolution during sintering of blended elemental Ti-5Al-2.5Fe alloy, J. Alloy Compd., 476 (2009) 130-137.

[13] R. Yamanoglu, W. Bradbury, E. A. Olevsky, R. M. German, Sintering and microstructure characteristics of $42 \mathrm{CrMo} 4$ steel processed by spark plasma sintering, Met Mater-Int. 19(5) (2013) 1029-1034.

[14] R. Yamanoglu, W. Bradbury, E. Karakulak, E. A. Olevsky, R. M. German, Characterisation of nickel alloy powders processed by spark plasma sintering, Powder Metall. 57 (2014) 380-386. 
[15] V. Medri, F. Monteverde, A. Balbo, A. Bellosi, Comparison of ZrZrC-SiC composites fabricated by spark plasma sintering and hot pressing, B2Adv Eng Mater. 7(3) (2005) 159163.

[16] A. Eldesouky, M. Johnson, H. Svengen, M. M. Attallah, H. G. Salem, Effect of grain size reduction of AA2124 aluminum alloy powder compacted by spark plasma sinterign, J. Alloy Compd., 609 (2014) 215-221.

[17] A. Ibrahim, F. Zhang, E. Otterstein, E. Burkel, Processing of porous Ti and Ti5Mn foams by spark plasma sintering, Mater Design. 32 (2011) 146-153.

[18] W. L. Bradbury, E. A. Olevsky, Production of SiC-C composites by free-pressureless spark plasma sintering (FPSPS) Scripta Mater. 63 (2010) 77-80.

[19] H. O. Gulsoy, N. Gulsoy, R. Calisici, Particle morphology influence on mechanical and biocompatibility properties of injection molded Ti alloy powder, Bio-Med Mater Eng. 24 (2014) 1861-1873.

[20] M. Guden, E. Celik, E. Akar, S. Cetiner, Compression testing of a sintered Ti6Al4V powder compact biomedical applications, Mater Charact. 54 (2005) 399-408.

[21] R. M. German, Sintering: From Empirical Observations to Scientific Principles, first ed., Oxford, Elsevier, 2014.

[22] P. S. Liu, G. F. Chen, Porous Materials, Processing and Applications, Tsinghua University Press Limited, Elsevier, 2014. 


\section{FIGURE CAPTIONS}

Figure 1. a) Die design used in the experiments, b) SEM image of the as-received pre-alloyed Ti5Al2.5Fe powders

Figure 2. a) Macro image of porous compacts processed via PSPS, b) SEM images of the porous compacts

Figure 3. Compression test results and the shear band produced during porous compact failure

Figure 4. SEM images of the surfaces of the sintered compacts: a) 7 days immersion, b) 14 days immersion, c) 21 days immersion in SBF, d) living cells on the sample surface after $96 \mathrm{~h}$ culture 

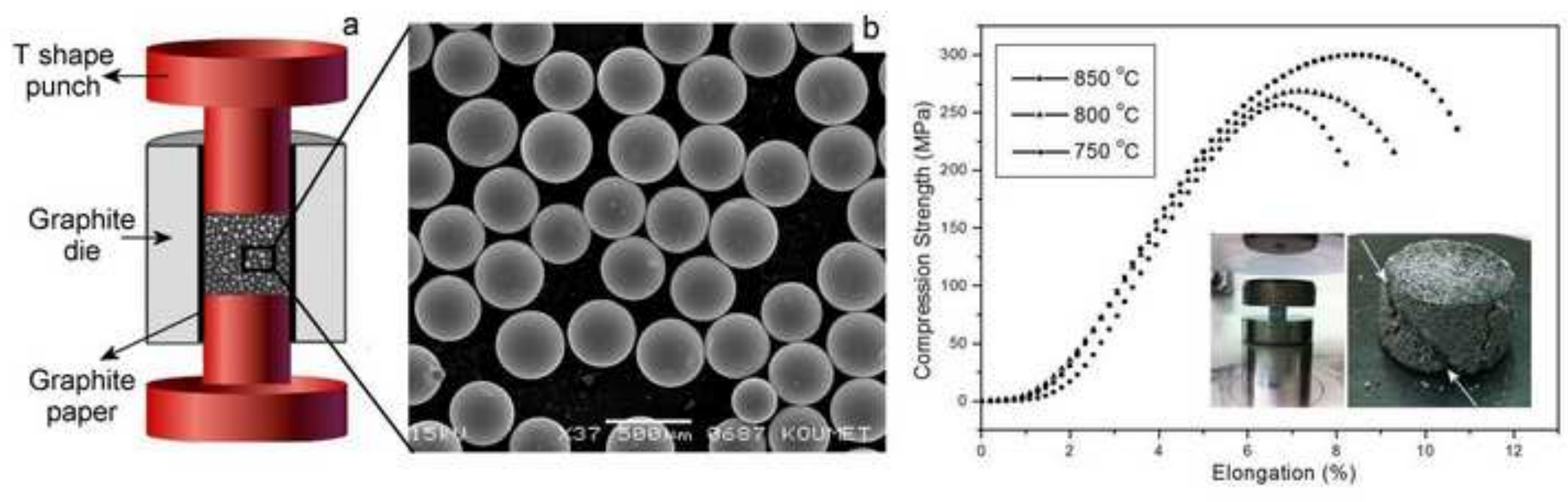


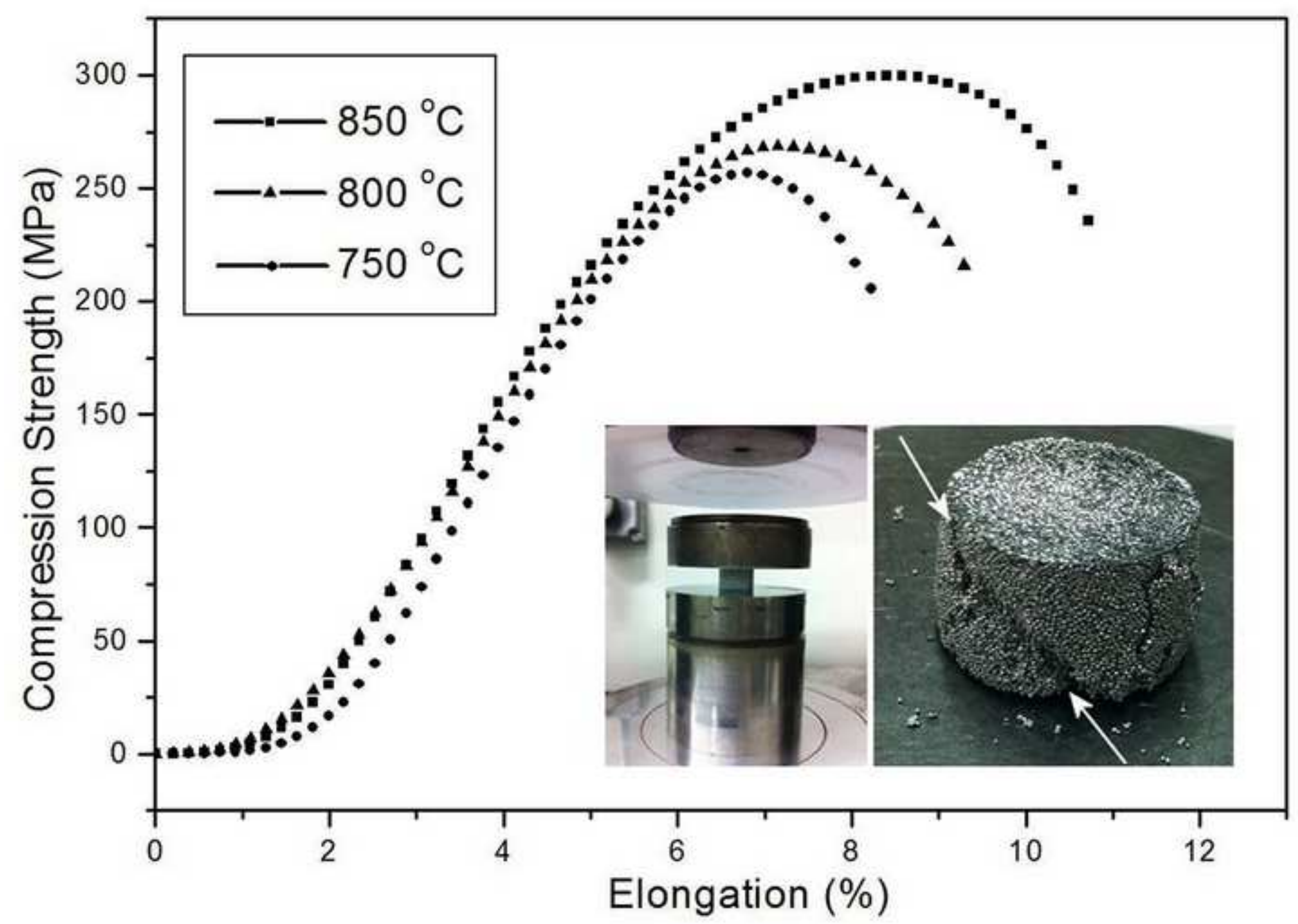


